\title{
REVISIÓN DE LITERATURA SOBRE LAS BARRERAS A LA TRANSFORMACIÓN DIGITAL Y SU RELACIÓN CON EL RENDIMIENTO FINANCIERO
}

\author{
RUBÉN AHOMED \\ Facultad de Comunicación, Universidad de Lima, Lima, Perú \\ aahomed@ulima.edu.pe \\ ORCID 0000-0002-7307-5375
}

\section{Resumen}

La transformación digital es una actividad que han venido adoptando las empresas para aprovechar los desarrollos tecnológicos digitales y mantenerse a la vanguardia empresarial rentablemente. El propósito del presente análisis es realizar una revisión de la literatura relacionada con la transformación digital, las barreras que existen para alcanzarla y su rendimiento financiero. A través de una revisión de 59 artículos, se definió un marco conceptual con nueve definiciones para la transformación digital, nueve para las barreras a la innovación y cinco para el rendimiento financiero. Los principales hallazgos son que existen barreras para el desarrollo de la transformación digital empresarial, pero difieren según la industria y país; por otro lado, el beneficio financiero no es claro y depende del contexto.

PALABRAS CLAVE: transformación digital / barreras / rentabilidad / rendimiento financiero

\section{Abstract}

\section{LITERATURE REVIEW ON BARRIERS TO DIGITAL TRANSFORMATION AND ITS RELATIONSHIP WITH THE FINANCIAL PERFORMANCE}

Digital transformation is an activity that companies have been adopting to take advantage of digital technology developments and stay at the business forefront profitably. The purpose of this analysis is to review the literature related to digital transformation, the barriers to achieving it and its financial performance. Through a review of 59 articles, a conceptual framework was defined with nine definitions for digital transformation, nine for barriers to innovation and five for financial performance. The main findings are that there are barriers to the development of the digital business transformation, but they differ according to the industry and country. On the other hand, the financial benefit is not clear and depends on the context.

KEYWORDS: digital transformation / barriers / profitability / financial performance 


\section{INTRODUCCIÓN}

Una empresa en proceso de transformación digital utiliza nuevas tecnologías digitales como los medios sociales, el acceso móvil, el análisis o los dispositivos integrados para permitir importantes progresos empresariales como la mejora de la experiencia del cliente, la racionalización de las operaciones o la creación de nuevos modelos empresariales (Singh y Hess, 2017). Sin embargo, se advierte que la transformación digital es un proceso de cambio, que no se enfoca en la tecnología, sino que se sirve de ella para lograr sus objetivos (Westerman, 2018). Asimismo, en un entorno VUCA (abreviación, por sus siglas en inglés, de volatility, uncertainty, complexity, ambiguity), en donde la transformación digital se desarrolla dentro de un estado de bloqueo, producto de la pandemia por el COVID 19, es imperativo que las empresas adopten modelos digitales de gestión para alcanzar un cierto nivel de madurez digital, puesto que empresas con mayor nivel de madurez, son generalmente más flexibles. Y, por el contrario, las empresas menos maduras son más frágiles (Fletcher y Griffiths, 2020).

\section{MARCO TEÓRICO-CONCEPTUAL: ESTADO DEL ARTE}

\subsection{Transformación digital}

La transformación digital es un proceso que tiene por objeto mejorar una empresa promoviendo cambios en sus características mediante la combinación de tecnologías de la información, la computación, la comunicación y la conectividad (Legner, Eymann, Hess, Matt, Böhmann, Drews, Mädche, Urbach y Ahlemann, F., 2017; Vial, 2019). Es la transformación de las actividades, los procesos, las competencias y los modelos empresariales para aprovechar los cambios y las oportunidades que traen consigo las tecnologías digitales (Demirkan, Spohrer y Welser, 2016). La transformación digital es una forma de transformación empresarial, facilitada por los sistemas de información, que va acompañada de cambios económicos y tecnológicos tanto a nivel organizativo como industrial (Chanias, Myers y Hess, 2019); dependiendo de la industria, el desarrollo de capacidades dinámicas gerenciales y organizacionales es clave para que su desarrollo sea exitoso (Li, Su, Zhang y Mao, 2017). Finalmente, para lograr una transformación digital exitosa, se tiene que trabajar con el personal de la empresa dándoles autonomía para crear o participar en equipos de la manera que deseen, incluso con socios externos (Westerman, Soule y Eswaran, 2019).

\subsection{Barreras a la innovación}

Las barreras son definidas como obstáculos a la innovación (Borins, 2001) o como factores que inhiben la aceptación de la tecnología (Cenfetelli y Schwarz, 2011). Asimismo, muchas empresas pueden tomar diferentes rutas para mejorar su rendimiento cuando la transformación digital cambia sus modelos de negocios (Bouwman, Nikou, y de Reuver, 2019). Bajo 
esta perspectiva, la inercia y la resistencia son las principales barreras organizacionales que limitan un proceso de transformación digital (Vial, 2019), aglutinando a muchas otras. Por ejemplo, en empresas de negocios electrónicos se encontraron cinco barreras para la adopción de innovación: a) percepción de riesgo, b) déficit de conocimiento, c) confianza, d) tamaño de la empresa y e) disposición organizativa (Johnson, 2010). En empresas con pocos años, se encontraron siete barreras: a) falta de financiación al interior de la empresa, b) falta de financiación de otras empresas, c) falta de personal calificado, d) falta de información en tecnología, e) dificultad para encontrar socios para innovación, f) mercado dominado por empresas establecidas y g) demanda incierta de bienes o servicios innovadores (Pellegrino, 2018). En educación, se encontraron cuatro barreras: a) el desafío de aprovechar la motivación intrínseca de los participantes, b) la creación de oportunidades de colaboración y comunicación entre los diferentes interesados, c) la búsqueda de tiempo suficiente para programar y aplicar una innovación y d) la falta de apoyo a los recursos digitales (Lanford, Corwin, Maruco y Ochsner, 2019).

\subsection{Rendimiento financiero}

El rendimiento financiero producto de la innovación digital es contradictorio y probablemente dependiente del contexto (Khin y Ho, 2019). Sin embargo, se encontró que las empresas con niveles de innovación digital superiores al promedio tienen mayores ingresos y márgenes de rentabilidad en 9 \% y $26 \%$ respectivamente (Westerman, Bonnet y McAfee, 2014). En una investigación hecha a 450 grandes empresas y una encuesta a 1559 personas en 106 países, se encontró que el 74 \% de las organizaciones no han podido establecer indicadores clave para ayudarlos a medir el impacto financiero de la transformación digital (Fitzgerald, Kruschwitz, Bonnet y Welch, 2013). En Rusia se halló que las empresas con mayor actividad digital obtienen mayores ingresos, beneficios y valor de mercado que aquellas con una actividad pasiva (Veselovsky, Izmailova, Yunusov, y Yunusov, 2019). En Malasia encontraron que la innovación digital medía el efecto de la orientación tecnológica y la capacidad digital en los resultados financieros y no financieros (Khin y Ho, 2019). En un estudio longitudinal hecho en Bélgica los periódicos de la zona flamenca siguen buscando rentabilidad al apostar por la reducción de costos y la expansión a pesar de la penetración de la digitalización de los contenidos (Van der Burg y Van den Bulck, 2017).

\section{METODOLOGÍA}

La revisión de la literatura se realizó utilizando la base de datos Web of Science (WoS), con la siguiente fórmula: digital transformation AND barriers; para ello se seleccionaron los artículos desde el año 2016 hasta agosto del 2020 obteniendo 89 artículos en 63 categorías. Se revisaron los artículos de las primeras diez categorías con un total de 
59 artículos que representó el $67 \%$ del total. Finalmente, se aplicó el criterio de definición de conceptos para escoger los artículos que explicaban detalladamente ambos constructos quedando nueve artículos por cada uno. Asimismo, se consideraron otros autores que fueron fuentes bibliográficas de varios de los autores que se investigó según los resultados en WoS, pero que no estaban dentro del criterio de tiempo que se usó para discriminar los años de evaluación de artículos.

\section{RESULTADOS}

Los resultados presentados a continuación se resumen en dos tablas con los conceptos de la transformación digital y las barreras a la innovación.

Tabla 1

Conceptos de transformación digital

\begin{tabular}{|c|c|}
\hline Autor & Concepto \\
\hline Vial (2019) & $\begin{array}{l}\text { Proceso que tiene por objeto mejorar una entidad desencadenando } \\
\text { cambios significativos en sus propiedades mediante combinaciones de } \\
\text { tecnologías de información, informática, comunicación y conectividad. }\end{array}$ \\
\hline
\end{tabular}

Demirkan, H., Spohrer, J. C. Es la transformación profunda y acelerada de las actividades, procesos, y Welser, J. J. (2016) competencias y modelos comerciales para aprovechar los cambios y oportunidades que traen consigo las tecnologías digitales y su impacto en toda la sociedad de manera estratégica y prioritaria.

Chanias, S., Myers, M. D. y Hess, T. (2019)

El uso extendido de la informática avanzada, como la analítica, la computación móvil, social media o dispositivos inteligentes incorporados y la mejora del uso de tecnologías tradicionales, como la planificación de los recursos empresariales (ERP), para permitir grandes mejoras en los negocios.

Li, L., Su, F., Zhang, W. y Mao, J. Y. (2017)

Andriole, S. (2017)

Singh, A. y Hess, T. (2017)

Westerman, G. (2018)

Fitzgerald, M., Kruschwitz, N., Bonnet, D. y Welch, M. (2013)

Legner et al. (2017)
La transformación digital pone de relieve el impacto de la tecnología de la información en la organización, estructura, rutinas y flujo de información.

La transformación digital no es una actualización de software o un proyecto de mejora en la cadena de suministro. Es un impacto digital planeado para lo que puede ser un sistema que funciona razonablemente.

Una empresa en proceso de transformación digital utiliza nuevas tecnologías digitales, como los medios sociales, el acceso móvil, el análisis o los dispositivos integrados para permitir importantes mejoras empresariales, como la mejora de la experiencia del cliente, la racionalización de las operaciones o la creación de nuevos modelos empresariales.

Es un proceso de cambio, que no se enfoca en la tecnología, sino que se sirve de ella para lograr sus objetivos.

El uso de nuevas tecnologías digitales (medios sociales, móviles, analíticas o dispositivos incorporados) para permitir importantes mejoras comerciales (como mejorar la experiencia del cliente, racionalizar las operaciones o crear nuevos modelos de negocio).

Describe los cambios impuestos por las tecnologías de la información (TI) como un medio para automatizar (parcialmente) las tareas. 
Tabla 2

Barreras a la innovación

\begin{tabular}{|c|c|}
\hline Autor & Concepto \\
\hline Johnson, M. (2010) & $\begin{array}{l}\text { a) Percepción de riesgo b) Déficit de conocimiento c) Confianza, d) Tamaño } \\
\text { de la empresa e) Disposición organizativa. }\end{array}$ \\
\hline $\begin{array}{l}\text { Fitzgerald, M., Kruschwitz, } \\
\text { N., Bonnet, D. y Welch, M. } \\
\text { (2013) }\end{array}$ & $\begin{array}{l}\text { a) Falta de urgencia b) Falta de visión c) Falta de dirección d) Actitud de los } \\
\text { trabajadores con mayor experiencia e) Tecnología tradicional f) Fatiga a la } \\
\text { innovación g) Política interna h) Abogar por el cambio i) Falta de incentivos. }\end{array}$ \\
\hline Pellegrino, G. (2018) & $\begin{array}{l}\text { a) Falta de financiación al interior de la empresa b) Falta de financiación } \\
\text { de otras empresas c) Falta de personal calificado d) Falta de informa- } \\
\text { ción en tecnología e) Dificultad para encontrar socios para innovación f) } \\
\text { Mercado dominado por empresas establecidas g) Demanda incierta de } \\
\text { bienes o servicios innovadores. }\end{array}$ \\
\hline $\begin{array}{l}\text { Lanford, M., Corwin, } \\
\text { Z. B., Maruco, T. } \\
\text { y Ochsner, A. (2019) }\end{array}$ & $\begin{array}{l}\text { a) El desafío de aprovechar la motivación intrínseca de los participantes } \\
\text { b) La creación de oportunidades de colaboración y comunicación entre } \\
\text { los diferentes interesados c) La búsqueda de tiempo suficiente para } \\
\text { programar y aplicar una innovación d) La falta de apoyo a los recursos } \\
\text { digitales. }\end{array}$ \\
\hline $\begin{array}{l}\text { Horváth, D. y Szabó, } \\
\text { R. Z. (2019) }\end{array}$ & $\begin{array}{l}\text { a) Recursos humanos y circunstancias de trabajo b) Escasez de recursos } \\
\text { financieros c) Problemas de estandarización d) Preocupación por la segu- } \\
\text { ridad cibernética y los problemas de propiedad de los datos e) Riesgo de } \\
\text { fragilidad f) Integración tecnológica g) Dificultad de coordinación entre las } \\
\text { unidades organizativas h) Falta de habilidades y actividades de planifica- } \\
\text { ción i) Resistencia organizativa. }\end{array}$ \\
\hline Vial, G. (2019) & a) Inercia b) Resistencia. \\
\hline $\begin{array}{l}\text { Cichosz, M.,Wallenburg, C. } \\
\text { M. y Knemeyer, A. M. (2020) }\end{array}$ & $\begin{array}{l}\text { a) Complejidad de la red logística (en el negocio de los servicios logísticos) } \\
\text { b) Falta de recursos. }\end{array}$ \\
\hline $\begin{array}{l}\text { Agrawal, P., Narain, R. } \\
\text { y Ullah, I. (2020) }\end{array}$ & $\begin{array}{l}\text { a) Alto sentido de urgencia b) Falta de directrices específicas para la } \\
\text { industria c) Falta de habilidades y talento digital d) Alto costo de imple- } \\
\text { mentación y funcionamiento. }\end{array}$ \\
\hline $\begin{array}{l}\text { Stentoft, J., Adsbøll } \\
\text { Wickstrøm, K., Philipsen, K. } \\
\text { y Haug, A. (2020) }\end{array}$ & $\begin{array}{l}\text { a) Falta de conocimiento sobre las nuevas tecnologías digitales b) } \\
\text { Falta de normas c) Más atención a la operación a expensas del desa- } \\
\text { rrollo de la empresa d) Falta de protección de datos (ciberseguridad) e) } \\
\text { Falta de personal calificado f) Falta de preparación de los empleados g) } \\
\text { Requerimiento de la educación continua de los empleados h) Falta de } \\
\text { comprensión de la importancia estratégica de las nuevas tecnologías } \\
\text { digitales i) Falta de comprensión de la interacción entre la tecnología y los } \\
\text { seres humanos j) Pocos recursos financieros k) Pocos recursos humanos } \\
\text { (mano de obra). }\end{array}$ \\
\hline
\end{tabular}

Elaboración propia

\section{DISCUSIÓN}

Se encontró una variedad de definiciones del concepto de transformación digital vinculados a procesos, competencias y modelos comerciales, relacionados con la informática 
o al impacto de la tecnología de la información. Por otro lado, existe una variedad de barreras dependiendo de las industrias y los países pero que se resumen dentro de los conceptos de inercia y resistencia. Finalmente, los resultados financieros son aún difíciles de identificar (Fitzgerald et al., 2013); sin embargo, existe evidencia de que es positiva, no obstante, siguen prácticas tradicionales para encontrar el beneficio.

\section{CONCLUSIONES}

La transformación digital empresarial en un proceso que se viene implementando hace varios años, su objetivo fundamental es llevar a las organizaciones a un estado que haga que sus negocios se mantengan rentables. Para lograr esto se valen del uso de la tecnología digital en sus diferentes manifestaciones, superando una serie de barreras. Sin embargo, encontrar el rendimiento financiero sigue siendo una labor compleja de lograr puesto que no se cuenta con el desarrollo correcto de los indicadores o en otros casos depende del contexto en que se desarrolla la empresa. Finalmente, en el entorno VUCA de la actualidad, las empresas tienen que buscar aceleradamente la digitalización de sus negocios, puesto que les proveerá de la agilidad necesaria para hacer frente a los cambios producto de factores exógenos como el del COVID 19.

\section{REFERENCIAS}

Agrawal, P., Narain, R. y Ullah, I. (2020). Analysis of Barriers in Implementation of Digital Transformation of Supply Chain Using Interpretive Structural Modelling Approach. Journal of Modelling in Management, 15(1), 297-317. https://doi. org/10.1108/JM2-03-2019-0066

Andriole, S. (2017). Five Myths About Digital Transformation. MIT Sloan Management Review, 58(3), 20-22.

Borins, S. (2001). Innovation, Success and Failure in Public Management Research: Some Methodological Reflections. Public Management Review, 3(1), 3-17. https://doi. org/10.1080/14616670010009423

Bouwman, H., Nikou, S. y de Reuver, M. (2019). Digitalization, business models, and SMEs: How do business model innovation practices improve performance of digitalizing SMEs? Telecommunications Policy, 43(9), 101828. https://doi.org/10.1016/j. telpol.2019.101828

Cenfetelli, R. T. y Schwarz, A. (2011). Identifying and Testing the Inhibitors of Technology Usage Intentions. Information Systems Research, 22(4), 808-823. https://doi. org/10.1287/isre.1100.0295 
Chanias, S., Myers, M. D., y Hess, T. (2019). Digital Transformation Strategy Making in Pre-Digital Organizations: The Case of a Financial Services Provider. Journal of Strategic Information Systems, 28(1), 17-33.

Cichosz, M., Wallenburg, C. M. y Knemeyer, A. M. (2020). Digital Transformation at Logistics Service Providers: Barriers, Success Factors and Leading Practices. International Journal of Logistics Management, 31(2), 209-238.

Demirkan, H., Spohrer, J. C. y Welser, J. J. (2016). Digital Innovation and Strategic Transformation. IT Professional, 18(6), 14-18. https://doi.org/10.1287/isre. 1100.0295

Fitzgerald, M., Kruschwitz, N., Bonnet, D. y Welch, M. (2013). Embracing Digital Technology: A New Strategic Imperative. MIT Sloan Management Review, 55(2), 1-12.

Fletcher, G. y Griffiths, M. (2020). Digital Transformation During a Lockdown. International Journal of Information Management, ahead-of-p(ahead-of-print). https://doi. org/10.1016/j.ijinfomgt.2020.102185

Horváth, D. y Szabó, R. Z. (2019). Driving Forces and Barriers of Industry 4.0: Do Multinational and Small and Medium-Sized Companies Have Equal Opportunities? Technological Forecasting and Social Change, 146, 119-132. https:// doi.org/10.1016/j.techfore.2019.05.021 h

Johnson, M. (2010). Barriers to Innovation Adoption: A Study of E-Markets. Industrial Management \& Data Systems, 110(2), 157-174. https://doi.org/10.1108/02635 571011020287

Khin, S. y Ho, T. C. F. (2019). Digital Technology, Digital Capability and Organizational Performance: A Mediating Role of Digital Innovation. International Journal of Innovation Science, 11(2), 177-195. h https://doi.org/10.1108/IJIS-08-2018-0083

Lanford, M., Corwin, Z. B., Maruco, T. y Ochsner, A. (2019). Institutional Barriers to Innovation: Lessons From a Digital Intervention for Underrepresented Students Applying to College. Journal of Research on Technology in Education, 51(3), 203-216. https://doi.org/10.1080/15391523.2019.1576558 ps

Legner, C., Eymann, T., Hess, T., Matt, C., Böhmann, T., Drews, P., Mädche, A., Urbach, N. y Ahlemann, F. (2017). Digitalization: Opportunity and Challenge for the Business and Information Systems Engineering Community. Business \& Information Systems Engineering, 59(4), 301-308. https://doi.org/10.1007/s12599-017-0484-2

Li, L., Su, F., Zhang, W. y Mao, J. Y. (2017). Digital Transformation by SME Entrepreneurs: A Capability Perspective. Information Systems Journal, 28(6), 1129-1157. 
Pellegrino, G. (2018). Barriers to Innovation in Young and Mature Firms. Journal of Evolutionary Economics, 28(1), 181-206. https://doi.org/10.1007/s00191-017 $-0538-0$

Singh, A. y Hess, T. (2017). How Chief Digital Officers Promote the Digital Transformation of their Companies. MIS Quarterly Executive, 16(1), 1-17.

Stentoft, J., Adsbøll Wickstrøm, K., Philipsen, K. y Haug, A. (2020). Drivers and Barriers for Industry 4.0 Readiness and Practice: Empirical Evidence from Small and Medium-Sized Manufacturers. Production Planning and Control, 1-18. https://doi. org/10.1080/09537287.2020.1768318

Van der Burg, M. y Van den Bulck, H. (2017). Why are Traditional Newspaper Publishers Still Surviving in the Digital Era? The impact of Long-Term trends on the Flemish Newspaper Industry's Financing, 1990-2014. Journal of Media Business Studies, 14(2), 82-115. https://doi.org/10.1080/16522354.2017.1290024

Veselovsky, M. Y., Izmailova, M. A., Yunusov, L. A., y Yunusov, I. A. (2019). Quality of Digital Transformation Management on the Way of Formation of Innovative Economy of Russia. Quality - Access to Success, 20(169), 66-71.

Vial, G. (2019). Understanding Digital Transformation: A Review and a Research Agenda. Journal of Strategic Information Systems, 28(2), 118-144. https://doi.org/10.1016/j. jsis.2019.01.003

Westerman, G. (2018). Your Company Doesn't Need a Digital Strategy. MIT Sloan Management Review, 59(3), 1-5.

Westerman, G., Bonnet, D. y McAfee, A. (2014). Leading Digital: Turning Technology into Business Transformation. Boston, MA: Harvard Business Review Press.

Westerman, G., Soule, D. L. y Eswaran, A. (2019). Building Digital Ready Culture in Traditional Organizations. MIT Sloan Management Review, 60(4), 59-68. 